\title{
The Planning of Reducing the Train Reception of Angular Traffic Flows in Bidirectional Marshalling Yard
}

\author{
Qing Sun ${ }^{1, a}$ Jian $\mathrm{Li}^{1, \mathrm{~b}}$ \\ ${ }^{1}$ School of Traffic and Transportation, Beijing Jiaotong University, China \\ a15125782@bjtu.edu.cn, ${ }^{\mathrm{b}} 12114230 @$ bjtu.edu.cn
}

\begin{abstract}
Keywords: marshalling station; angular traffic flow; train reception plan; binary tree
Abstract. The angular traffic flows of the bidirectional marshalling station would greatly increase the task for its characteristic of repeated unmarshalling with subsequently affecting the productive efficiency. Therefore, how to decrease the amount of trains of angular remains an urgent problem needed to be solved. The paper is constructed of a mathematical model of the train reception from the work-distribution of each yard and in each technical operating link, and regards the alternative arrival system as a binary tree problem while planning to reach its algorithm. The paper then draws a planning capable of limiting the fewest of reception trains of angular traffic flow. The paper sets Nanxiang marshalling station as a proof and a reference for the dispatchers.
\end{abstract}

\section{Introduction}

The angular traffic flow is the transit car with resorting whose arrival and departure destination are not in the same system that has to transit from one system to another. Angular traffic flow increases greatly the wasted journey of trains as well as the detention time at station, which would have a disadvantageous influence on the function of the station. What's more, it would arouse cross-interference with other operation and other unsafe factors. The research shows that when the angular traffic flow accounts for less than 15 percent of transit car with resorting and it has little effect on the capability of the marshalling station, but the waste would increase when the percentage is above 15 percent .

At present, there are several main optimizing strategies to effectively reduce angular traffic flows. Technical conditions permitting in both up and down systems, reverse train should be promoted to start up in order to exchange traffic flow on site. If it is in transport intersection and the station track is allowed to run. Along a direction, when the proportion of angular exchange traffic flow to the arrivals to be sorted is too large to all, reversing trains is supposed to be adopted, which means send the train into another system to go marshalling. Though the exchanged flows may increase partly, the whole exchange flows are cut down. The detailed measurement of the reduction of angular traffic flows in every marshalling station varies and many factors should be taken into consideration according to the main functions of each marshalling station in the rail network and its station features. By contrast, with the existing equipment and facilities, a proper selection of arrival system of disintegrating trains can radically reduce the number of angular traffic flows, and the workload of the technical operation of the marshalling station so as to improve the work efficiency of the marshalling station.

\section{Problem Description and Analysis}

The problem of the arrival and unmarshalling of trains at bidirectional marshalling station aimed at reducing the angular traffic is presented as follows: there are two systems of trains' arrival and unmarshalling, $k=0,1$, any train that arrives waiting to be sorted has its track direction $i(i=1,2, \ldots m)$, if the marshalling arrangement is clearly known, the amount of angular traffic flows varies according if different system is chosen. Therefore, it is vital to ensure the reception plan of the fewest angular traffic flow meet every rule.

Actually, the number of trains was set as $n(n=1,2, \ldots, j \ldots n)$ according to station shift operating plan. There are two kinds of trains, empty trains and loaded trains. While the former would not be included in the angular traffic flows, the direction of the latter is set as $i$ the same as that of arrival, but 
there are all transit cars with resorting in the arrangement, then there are possibly n- 1 directions the trains drive up to(except marshalling violations). If three or more directions connecting tracks exist, there would inevitably be angular traffic flows. Finally, the loaded trains in the arrangement would start out from the subsystem. Suppose the amount of trains is $a+b, a$ refers to the number of trains in the up-line system, $b$ refers to that of down system, then on choosing the direction of arrival, the amount is putting $a$ or $b$ into angular traffic flows. However, you may choose the less one without considering train track storing. Since every time after trains' arrival, technical operation will begin, which means the received trains will have an effect on their condition when they arrived at the yard. It should be judged according to time whether or not it will influence the next train When the technical arrival work is completed, the train be disintegrated by the shunting locomotive and finally be placed in marshalling yard tracks correspondingly.

Problem inputting: the arrival time of trains break up, marshalling arrangement, the operation capability of the train arrival and disintegration and the time of relevant technical work.

Problem output: the reception yard of trains in the marshalling station, the amount of angular traffic flows under the arrival plan.

\section{Build mathematical model}

Parameter Definition. Do not number your paper: Suppose $k$ to be the serial number of the bidirectional marshalling station, the number 0 represents up-line system, and 1 represents downward system; $T_{\text {daily }}$ means the arranged end time of train. The set of all the lines of marshalling yard $M=\{1,2,3 \ldots, \mathrm{m}\}$, here $m$ means the number of directions; $n$ means the amount of trains arrived break up, and $j$ the number of them, $i$, $i$ ' represents the directions of lines connecting marshalling station. $i \in M, i^{\prime} \in M$ and $i \neq i^{\prime} ; n_{d}^{k}$ represents the amount of tracks at the arriving yard in system $k, F_{i j i}$ the amount of train $j$ received from $i$ and leaving for $i$ ', and $D_{i i^{\prime}}^{k}$ all the cost in the operation of transiting yard. $T_{\text {inspection }}^{k}$ and $T_{b r e a k-u p}^{k}$ represent the standard time of train inspection and humping part in $k$ system, $t_{(j)}^{d}$ the arrival time of $j$ and $t(j)$ the actual time of arrival. $\Delta t$ means additional waiting time of $j$ when the lines are full.

Definition of decision variables:

$x_{i j}^{k}, y(j), I_{j^{\prime}}^{k}$ —Boolean decision variables

$x_{i j}^{k}=\left\{\begin{array}{l}1, \text { The } j \text { train connected to the } k \text { system in the direction } i \\ 0, \text { Others }\end{array}\right.$

$y(j)=\left\{\begin{array}{l}1,1, \text { The added time needed by the } j \text { train during waiting } \\ 0, \text { Others }\end{array}\right.$

$I_{j^{\prime}}^{k}=\left\{\begin{array}{l}1, \text { The } j^{\prime} \text { train still has station track occupied after being connected into the } k \text { system } \\ 0, \text { Others }\end{array}\right.$

Model Construction. If the contents of trains arrived at bidirectional marshalling yard is settled, the trains could be classified according to their the lines of direction to arrive and departure; if they can be connected with both up-line and downward system, then there's another choice of arrival system. The angular trains would be different when trains arrive at different arrival yard. Its cost differs as well owing to the difference of running time and the distance. The mathematical model with restrictions of arrival and marshalling capability is presented as follows.

$$
\begin{aligned}
\min Z= & \sum_{k=0,1} \sum_{i \in M} \sum_{i^{\prime} \in M} \sum_{j=1}^{n} F_{i j i^{\prime}} x_{i j}^{k} D_{i i^{\prime}}^{k} \\
\text { s.t. } \quad & 0 \leq T=t(j) \leq T_{\text {class }} \quad \forall j \in 1,2 \ldots, n \\
& t(j)=t_{j}^{d}+y(j) \Delta t
\end{aligned}
$$




$$
\begin{aligned}
& y(j)= \begin{cases}0 & f(k, j) \leq n_{d}^{k} \\
1 & f(k, j)>n_{d}^{k}\end{cases} \\
& \Delta t=\min \left\{I_{j^{k}}^{k} t\left(j^{\prime}\right)+T_{\text {inspection }}^{k}+T_{\text {break-up }}^{k}-t_{j}^{d}\right\}
\end{aligned} \begin{aligned}
& f(k, j)=\sum_{0<j^{\prime}<j} I_{j^{\prime}}^{k} \leq n_{d}^{k} \\
& I_{j^{\prime}}^{k}= \begin{cases}0 & t(j)-t\left(j^{\prime}\right) \leq T_{\text {inspection }}^{k}+T_{\text {break-up }}^{k} \\
1 & t(j)-t\left(j^{\prime}\right) \leq T_{\text {inspection }}^{k}+T_{\text {break-up }}^{k}\end{cases} \\
& \sum_{k=0,1} x_{i j}^{k}(T)=1 \quad T \in\left(t(j), t(j)+T_{\text {inspection }}^{k}+T_{\text {break-up }}^{k}\right) \\
& \sum_{k=0,1} x_{i j}^{k}=1 \quad \forall i, j
\end{aligned}
$$

(9)

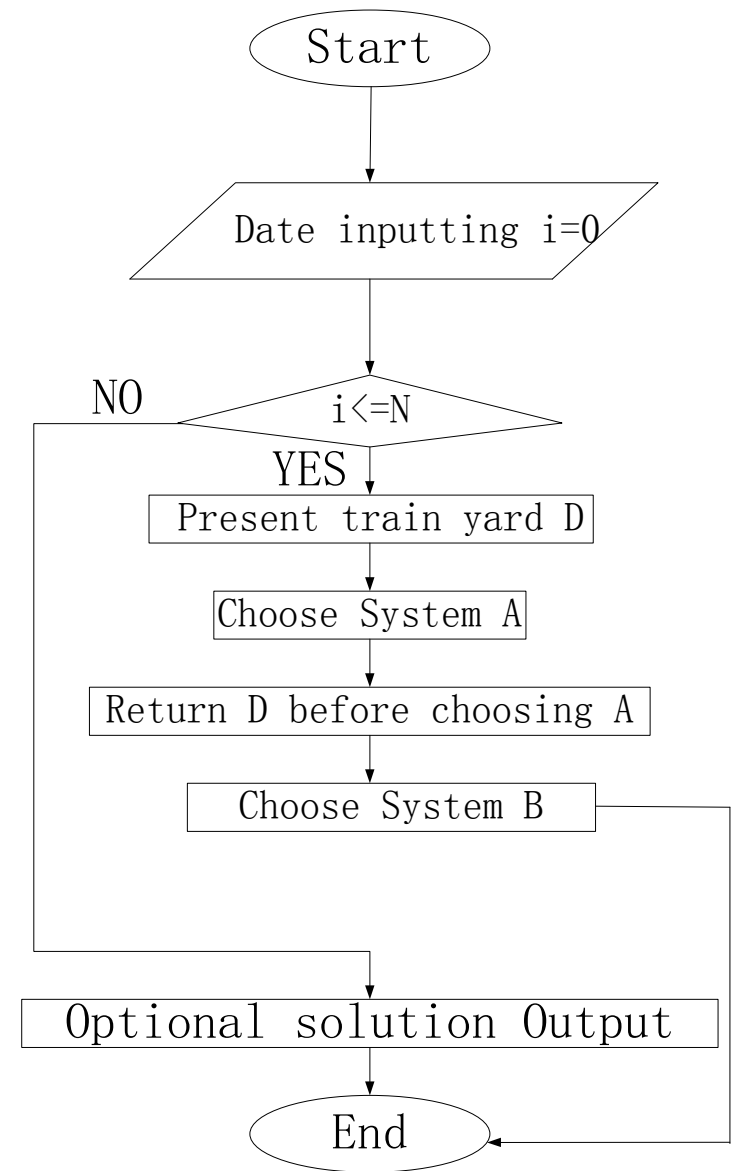

Figure 1. Algorithm Flow Chart

Hereby (1) is the target function, which is the minimum of angular train cost after the trains arrive according to plan; (2) represents planning time restrictions; (3) (5)shows that trains have to wait additional time because of tracks of arrival yard are all in operation. Here the additional time in (5) is the time lag of the expected arrival time of $j$ and the earliest time $j$ ' completes its humping work. (6) represents track constraints of arrival yard, (7)that of inspection and marshalling , (8) that of track occupying, and (9) the constraints of variable.

\section{Algorithm for solution}

Based on the above explanation, choosing other arrival system of each train is considered as a key problem in step n. Each decision will impact the following decision for it has aftereffect. Set the initial state of train yard within the scheduled time, first consider the present train yard state when 
before making a choice of the train reception, then add up the angular traffic flows until the plan is completed, finally the feasible plan that has the fewest angular flows. Since there are only two choice for the arrived train in their process of marshalling, and can be equal to the decision problems in $n$ steps, which is exactly consistent with the binary problem in computer data structure. Taking binary tree as a strategy of solution and Nanxiang as a field to study doesn't mean both of the directions can be connected with two systems, but an imperfect binary tree problem.

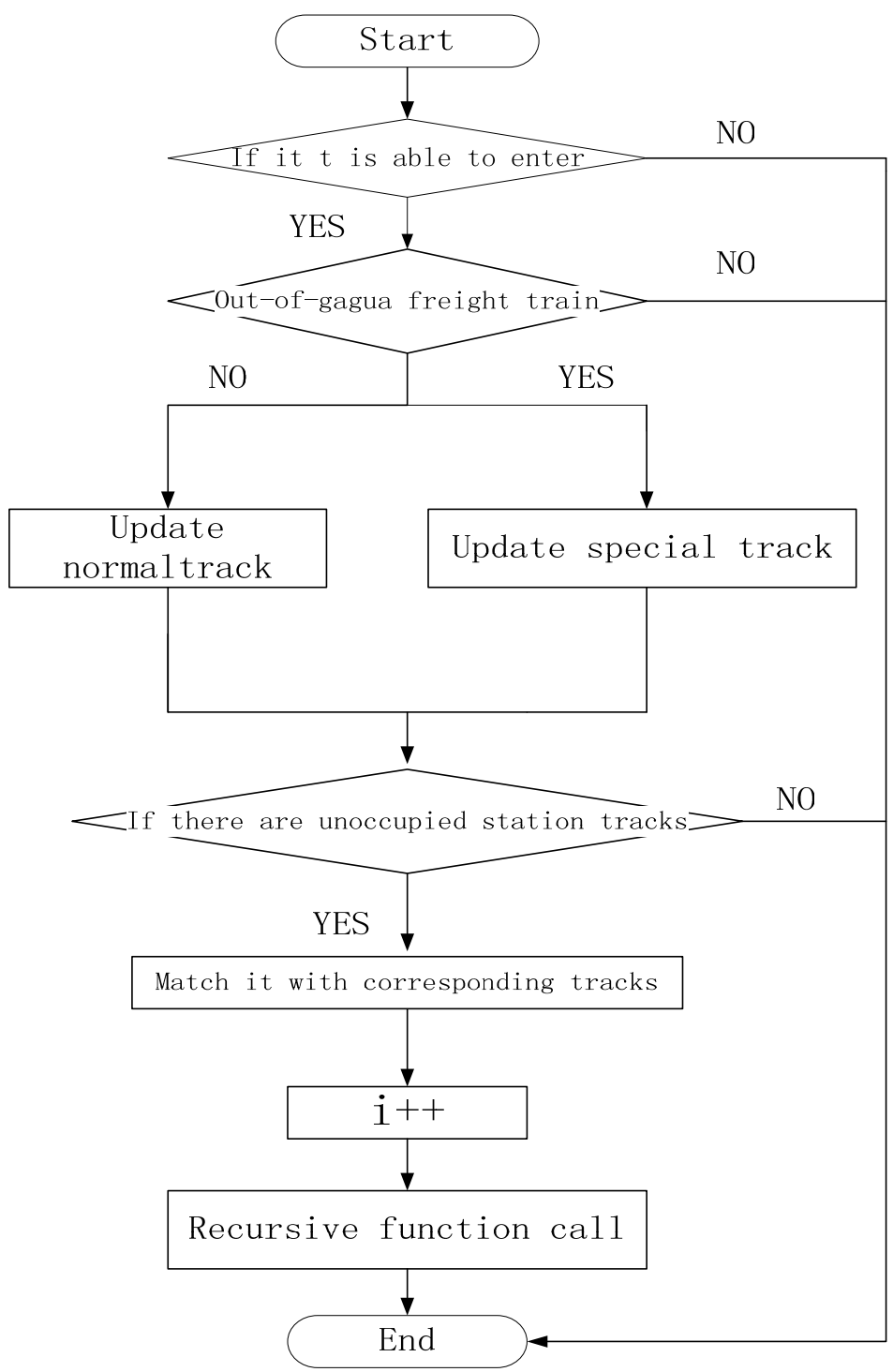

Figure 2 .A Explanation of Specific Process

It should be calculated according to concrete problems, which means it is necessary to judge if a train is out of gauge, and before the arrival of trains, the state of tracks in every arrival yard of system needs reassured with the recursive algorithm of preorder traversal of the binary trees, it is much more difficult to explain the algorithm clearly by word since there are many branches and circulation operations. The Figure 1 is the flow chart of algorithm, when the program begins, first input the arrival time of train and its marshalling contents, and then judge if the current sequence number is greater than the amount of trains, finally output the optimal solution and their corresponding scheme. Otherwise, choose system A to deal with the reception, the specific flow chart is shown in the Figure 2. According to the characteristics of recursive algorithm, if the binary tree is not empty, first traverse root node, next the left subtree, and finally the right subtree. Afterward, copy the arrival information and put it in system B to process before it gets process in A system. But since the parameter of A and $\mathrm{B}$ are different, the result differs. When all optimal solution is output after the traversal at the end of the program. 
Explanation of recursive function: it means try_next_state function, to begin with, compare $i$ with $\mathrm{N}$, if $i \geq N$, compare the angular traffic flows in present state with that in optimal state. If the former is smaller, update the optimal solution; otherwise, update the information of present state and try to enter into the reception work of the next train. Pass the information to try_next_state for the next recursive calculating.

\section{Analysis of examples.}

The study is based on the actual layout of Shanghai Nanxiang marshalling yard. Considering the calculating only includes transit loaded cars with resorting and needs the disposal data simplified on the basis of the marshalling arrangement of present arrival plan, the study selected the inputting data from the marshalling arrangement prediction (loaded) of arrival arrangement at a daytime marshalling station on a certain day. It is of bidirectional longitudinal type marshalling station with three levels and five yards, connecting three directions, Beijing-Shanghai line, Shanghai-Kunming line, and Nanxiang-HeJiawan line. The up-line yard has eight station tracks, among which 4 tracks can receive out-of-gauge freight train. It can receive the arrival train to be torn down from Beijing-Shanghai line, and Shanghai-Kunming line. The trains from Nanxiang-HeJiawan line needn't inspecting. It only needs to wait 30 minutes to get its piping picked up.The downward arriving yard has 10 train tracks and 4 of them can receive out-of-gauge freight train. It can receive arrival disintegrating trains from Beijing-Shanghai line and from Shanghai-Kunming line. The settled time of disintegrating work at marshalling station is 45 minutes, the humping pushing needs 25 minutes. A shift consists of 12 hours with the daytime from 6am to 6pm and night shift 6pm to 6am. The miles it runs are about the same within the marshalling station whether it runs from the up-line yard or downward yard. So the cost of fees transition is set as 1 . The study analyzes the train data of Nanxiang marshalling station, input the marshalling arrangement of a train (loaded trains) finally the result is presented in the Table 1 , finally calculated by $\mathrm{VC}++6.0$.

Table 1 Program output

\begin{tabular}{|c|c|c|c|c|c|}
\hline \multicolumn{6}{|c|}{ Angular Traffic Flows: 131 cars A: downward system B: up-line system } \\
\hline NO. & Arrival time & contents & Out-of-gauge & direction & Arrival system \\
\hline 0 & $7: 29$ & $35 / 17 / 52$ & $\mathrm{~N}$ & $\mathrm{HK}$ & $\mathrm{A}$ \\
\hline 1 & $7: 58$ & 3/5/8 & $\mathrm{S}$ & $\mathrm{NH}$ & $\mathrm{B}$ \\
\hline 2 & 8:17 & $12 / 4 / 16$ & $\mathrm{~N}$ & $\mathrm{NH}$ & $\mathrm{B}$ \\
\hline 3 & $8: 22$ & $43 / 1 / 44$ & $\mathrm{~S}$ & HK & A \\
\hline 4 & $8: 36$ & 4/2/6 & $\mathrm{N}$ & $\mathrm{NH}$ & B \\
\hline 5 & 8:39 & $48 / 0 / 48$ & $\mathrm{~N}$ & HK & A \\
\hline 6 & $8: 55$ & 8/0/8 & $\mathrm{N}$ & $\mathrm{NH}$ & B \\
\hline 7 & 9:18 & $14 / 16 / 30$ & $\mathrm{~S}$ & HK & B \\
\hline 8 & 9:35 & $21 / 16 / 41$ & $\mathrm{~N}$ & $\mathrm{NH}$ & A \\
\hline 9 & $9: 36$ & $43 / 2 / 45$ & $\mathrm{~N}$ & HK & A \\
\hline 10 & $10: 17$ & $11 / 1 / 12$ & $\mathrm{~N}$ & $\mathrm{NH}$ & B \\
\hline 11 & $10: 33$ & $23 / 2 / 25$ & $\mathrm{~N}$ & HK & A \\
\hline 12 & $10: 44$ & $11 / 1 / 12$ & $\mathrm{~N}$ & $\mathrm{NH}$ & B \\
\hline 13 & $10: 49$ & $1 / 13 / 14$ & $\mathrm{~N}$ & $\mathrm{JH}$ & A \\
\hline 14 & $10: 59$ & $22 / 3 / 25$ & $\mathrm{~S}$ & $\mathrm{JH}$ & A \\
\hline 15 & $11: 14$ & $12 / 3 / 15$ & $\mathrm{~N}$ & $\mathrm{JH}$ & A \\
\hline 16 & $11: 25$ & $22 / 0 / 22$ & $\mathrm{~N}$ & $\mathrm{NH}$ & B \\
\hline 17 & $11: 38$ & $16 / 6 / 22$ & $\mathrm{~N}$ & $\mathrm{NH}$ & B \\
\hline 18 & $11: 46$ & 5/9/14 & $\mathrm{N}$ & $\mathrm{JH}$ & A \\
\hline 19 & $12: 03$ & $6 / 3 / 9$ & $\mathrm{~N}$ & $\mathrm{JH}$ & A \\
\hline 20 & $12: 22$ & 13/2/15 & $\mathrm{N}$ & NH & B \\
\hline
\end{tabular}




$\begin{array}{lccccc}21 & 12: 39 & 23 / 17 / 40 & \mathrm{~N} & \mathrm{HK} & \mathrm{A} \\ 22 & 12: 51 & 12 / 30 / 42 & \mathrm{~N} & \mathrm{JH} & \mathrm{A} \\ 23 & 13: 14 & 45 / 0 / 45 & \mathrm{~N} & \mathrm{HK} & \mathrm{A} \\ 24 & 13: 46 & 11 / 0 / 11 & \mathrm{~N} & \mathrm{NH} & \mathrm{B} \\ 25 & 14: 05 & 10 / 8 / 18 & \mathrm{~S} & \mathrm{NH} & \mathrm{B} \\ 26 & 14: 11 & 0 / 25 / 25 & \mathrm{~N} & \mathrm{JH} & \mathrm{A} \\ 27 & 14: 14 & 15 / 3 / 18 & \mathrm{~N} & \mathrm{HK} & \mathrm{A} \\ 28 & 14: 21 & 26 / 12 / 38 & \mathrm{~N} & \mathrm{JH} & \mathrm{A} \\ 29 & 14: 30 & 15 / 16 / 31 & \mathrm{~S} & \mathrm{JH} & \mathrm{A} \\ 30 & 14: 32 & 48 / 0 / 48 & \mathrm{~N} & \mathrm{HK} & \mathrm{A} \\ 31 & 15: 19 & 36 / 0 / 36 & \mathrm{~N} & \mathrm{HK} & \mathrm{A} \\ 32 & 15: 46 & 26 / 0 / 26 & \mathrm{~N} & \mathrm{NH} & \mathrm{A} \\ 33 & 16: 10 & 15 / 10 / 25 & \mathrm{~N} & \mathrm{HK} & \mathrm{B} \\ 34 & 16: 16 & 8 / 1 / 9 & \mathrm{~N} & \mathrm{NH} & \mathrm{A} \\ 35 & 16: 25 & 25 / 30 / 55 & \mathrm{~N} & \mathrm{HK} & \mathrm{B} \\ 36 & 16: 35 & 9 / 0 / 9 & \mathrm{~N} & \mathrm{NH} & \mathrm{B} \\ 37 & 16: 39 & 34 / 2 / 36 & \mathrm{~N} & \mathrm{HK} & \mathrm{A} \\ 38 & 16: 41 & 35 / 8 / 43 & \mathrm{~N} & \mathrm{JH} & \mathrm{A} \\ 39 & 16: 49 & 7 / 10 / 17 & \mathrm{~N} & \mathrm{JH} & \mathrm{A} \\ 40 & 17: 00 & 4 / 3 / 7 & \mathrm{~N} & \mathrm{JH} & \mathrm{A} \\ 41 & 17: 04 & 1 / 8 / 9 & \mathrm{~N} & \mathrm{NH} & \mathrm{B} \\ 42 & 17: 14 & 46 / 0 / 46 & \mathrm{~N} & \mathrm{HK} & \mathrm{A} \\ 43 & 17: 40 & 10 / 0 / 10 & \mathrm{~N} & \mathrm{HK} & \mathrm{A}\end{array}$

Note:”NH” means Nanxiang-HeJiawan line, ”JH”shows Beijing-Shanghai line,”HK”means Shanghai-Kunming line.

In this mathematical problem, the parameter of the arrival plan is presented as follows: $\mathrm{k}=0$ is the up-line system, $\mathrm{k}=1$ is the downward system, $\mathrm{m}=3, T_{\text {daily }}=1800, n_{d}^{0}=8, n_{d}^{1}=10, T_{\text {break-up }}^{0,1}=25 \mathrm{~min}$ if the transit car with resorting needs to perform transiting operation, $D_{i i^{\prime}}^{k}=1$ otherwise, $D_{i i^{\prime}}^{k}=0$.

Through the process of calculating, finally other choice is made in No.44 arrival train as the optimal plan in No.44 arrival train with the total amount of traffic flow of 131 comparing with the transit trains of 600 per day.(the total amount of empty trains) Besides, the transit cars with resorting that Nanxiang marshalling yard received accounts for $70 \%$ of the total amount, which proves that the result through the calculating can decrease the number of transit cars to some extent, and thus giving the arrival scheme of trains that has the least angular traffic flows and thus acting relatively as a reference considering the actual capacity of the arrival and unmarshalling of trains. To make clearer predictions of the traffic flows on site, it is feasible to mark out he empty transit cars with resorting in detail, thereby drawing a conclusion with much better reference. For example, say the train from Nanxiang-HeJiawan line has a strict requirement of the SQ double-deck empty loading vehicles. But in practice of transporting there should be some adjustment so as to meet the demand of empty cars.

\section{Conclusion}

The dissertation takes in the experience and methods of planning in marshalling, coming to an algorithm through the $\mathrm{VC}++$ programme and proves its effectiveness by the calculating of the actual data based on the study of Nanxiang station. With the marshalling station keeping increasing, the dissertation aims at offering as a reference and opinions in the layout of stage plan and the planning of marshalling yard transiting. The paper also draws a scheme that has the fewest angular traffic flows through computer simulation within the corresponding condition restraint. With the deepening of railway transportation and the higher requirement of transportation organizations, the adjustment of arrival system of trains at the marshalling yard is a more feasible way that can radically cut down the angular traffic flows. 


\section{Acknowledgements}

This work was financially supported by National Natural Science Foundation of China(51178031), the Fundamental Research Funds for the Central Universities(2014YJS069),and Research and development plan of science and technology of China Railway Corporation(2014J006-C).

\section{References}

[1] Hui-Ming Niu and An-Zhou Hu:Journal of Northern Jiaotong University, 1998, Vol.22(6), pp.38-42.

[2] Hui-Ming Niu and An-Zhou Hu:China Railway Science, 2003, Vol.24(2), pp.115-120.

[3] LENTINK.R.M,FLOOLE and P.J,KROON.I.G,et.al.:Planning in Intelligent systems,2003,pp.415-436.

[4] Hao-dong Li,Shi-wei He, Bao-hua WANG and Yong-sheng SHEN: Journal of the China Railway Society, 2011,Vol.33(8),pp.13-22.

[5] Quan-Shou Zhang:Journal of Northern Jiaotong University, 1989, Vol.13(4), pp.64-72. 